\title{
التعلم القائم على الويب وتنمية استخدام بعض تطبيقات الحوسبة السحابية
}

\section{إعلداد}

\author{
د/ منصور عبد الفتاح أحمد \\ مدرس تكنولوجيا التعليم \\ كلية التربية بقنا - جامعة جنوب الوادى \\ mansourmans@yahoo.com
}

\author{
أ. أد/عماد شوقى ملقى سيفين \\ أستاذ المناهج وطرق التدربس \\ كلية التربية بقنا- جامعة جنوب الوادي المبن \\ emad.sefeen@edu.svu.edu.eg
}

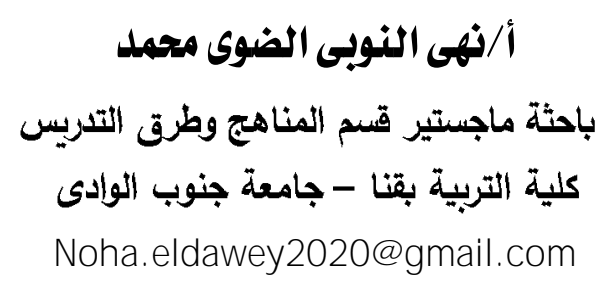




\section{التعلم القائم على الويب وتنمية استخلام بعض تطبيقات الحوسبة السحابية}

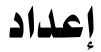

د/ منصور عبد الفتاح أحمد

مدرس تكنولوجيا التعليم

كلية التربية بقنا - جامعة جنوب الوادى

mansourmans@yahoo.com
أ. د.عماد شوقى ملقى سيفين

أستاذ المناهج وطرق التدربس

كلية التربية بقنا- جامعة جنوب الوادى التئي

emad.sefeen@edu.svu.edu.eg

\section{أ/زهى النوبى الضوى محمد}

باحثة ماجستير قسم المناهج وطرق التريس

كلية التربية بقنا - جامعة جنوب الوادي الونى

Noha.eldawey2020@gmail.com

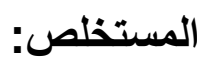

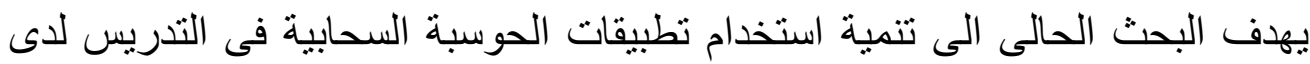

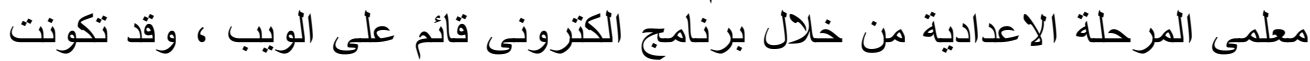

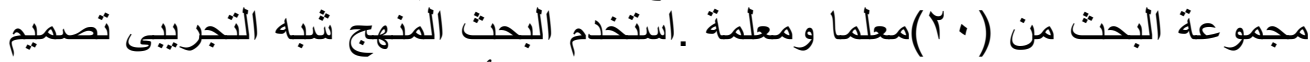

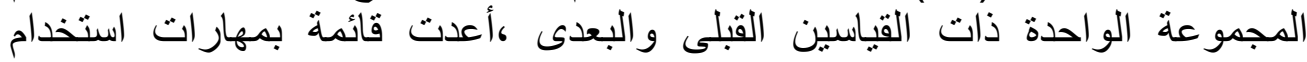

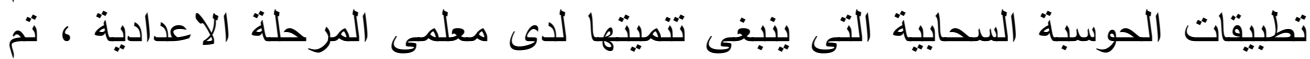

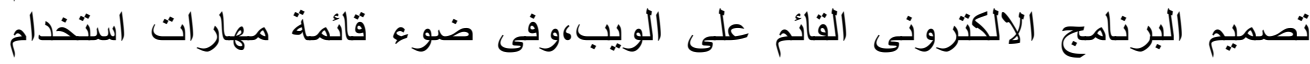

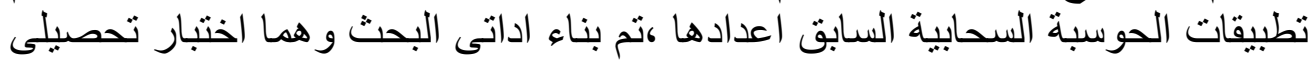

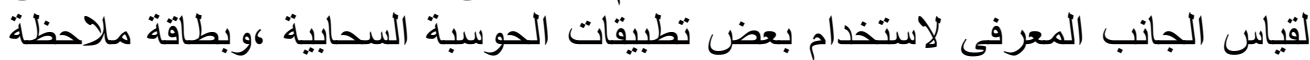

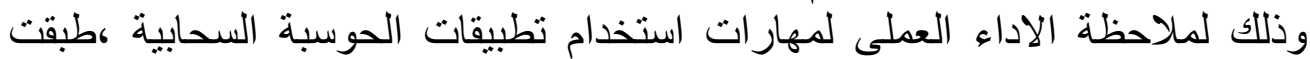

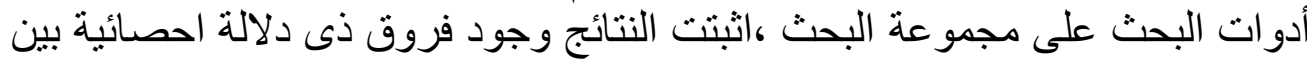

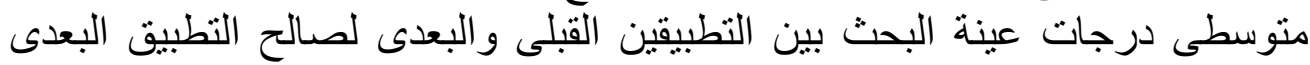

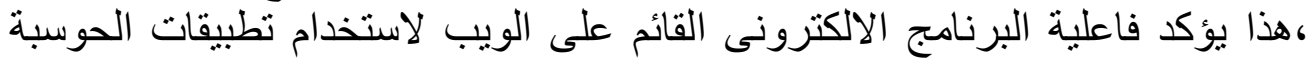

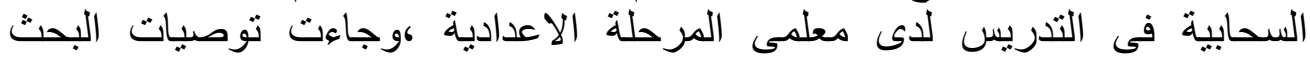

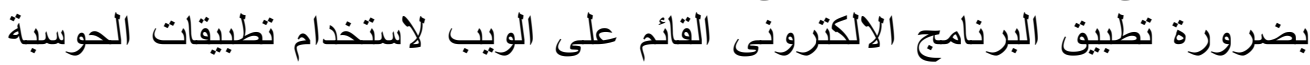

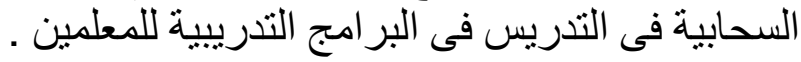
الكلمات المفتاحية : التعلم القائم على الويب ـ الحوسبة السحابية . 


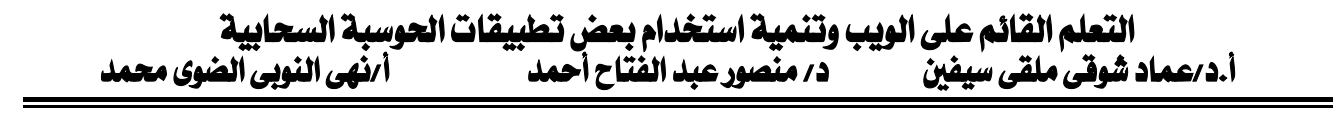

\section{A Web-based software learning and development of the use of some cloud computing application}

Prof. Dr. Emad Shawki Malky

Studying materials and teaching Methods

Faculty of Education in Qena,

South Valley University

emad.sefeen@edu.svu.
Dr. Mansour Abdel Fattah Ahmed

Education Technology Teacher

Faculty of Education in Qena

South Valley University

mansourmans@yahoo.com

\section{Noah El-Noubi El-Dawy Mohamed}

Researcher Master of Department of

Curriculum and Teaching Methods

Faculty of Education, Qena South Valley University

Noha.eldawey2020@gmail.com

\section{Abstract:}

The current research aims to use some cloud computing applications in teaching among middle school teachers through an electronic program based on the web, and the research group consisted of (20) teachers. The research used the quasi-experimental approach to designing the one group of pre and post measurements.A list of skills was prepared for using some cloud computing applications that should be developed by middle school teachers, The electronic program based on the web was designed, and in light of the list of skills for using some of the previously prepared cloud computing applications, The research tools were built and they are an achievement test to measure the cognitive aspect of the skills of using some cloud computing applications , And a note card to observe the practical performance of the skills of using some cloud computing applications, The research tools were applied to the research group, the results demonstrated that there are statistically significant differences between the average scores of the research sample Between the average scores of the research sample between the pre and post applications in favor of the post application, This confirms the effectiveness of the electronic program based on the web for the use of some cloud computing applications in teaching among middle school teachers, and research recommendations came to the necessity of applying the web based program to use some cloud computing applications in teaching at all educational levels.

Keywords: web-based learning - cloud computing 
يثهد العالم اتساع الفجوات بين من فى متناول ايديهم المعلومات والمعرفة، وبين من هم فى حاجة اليها، فهناك الكثيروالعديد من البشر يعيشون فى افريقيا وفى اثد الحاجة الى المعلومات والمعرفة ، ويعتبر التعليم و التدريب من اهم المقومات و المحاور الرئيسية للتنمية التكنولوجية .

كما ان المتعمين الان ينطلقوا بحرية لاستخدام الويب فى مختلف المجالات ومنتديات النقاش،وان تو افر التكنولوجيا الجديدة وظهور الحوسبة السحابية اعلى دقة فى الجيل القادم من التعلم الالكترونى، وان التعلم القائم على الويب يسمح بالوصول الى ئى

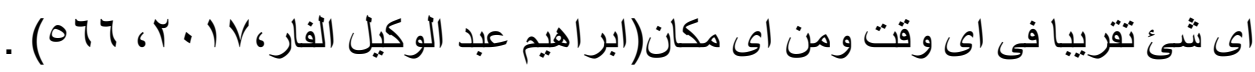

ويعد التعلم القائم على الويب احد مستحدثات تقنيات التعليم التى فرضت نفسها وبقوة على المنظومة التعليمية باعتبار ها بيئة تعلم تفاعلية عن بعد، بحيث يمكن للمتعلمين من خلالها المشاركة فى الار اء و التفاعل فيما بينهم وكأنهم داخل فصل واحد يعملون معا

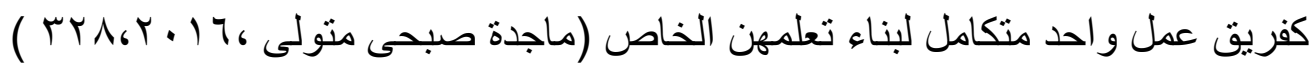

كما ان التعلم القائم على الويب يسهل وصول محتوى المقرر للمتعلم، ويعمل كوسيط للتفاعل بين المعلم وجميع طلابة،حيث تدعم التكنولوجيا الاتصسال من فرد لاخر، ومن

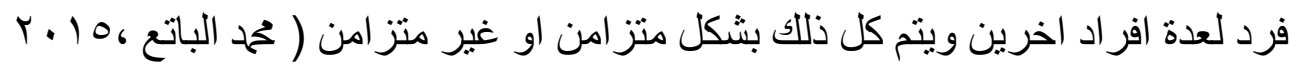

و الحوسبة Cloud Computing هى تكنولوجيا حديثة تعتمد على شبكة الانترنت السحابية

و الخوادم العملاقة ،ونوفر العديد من الخدمات والتطبيقات التى يدير ها مزود الخدمة ، وتمكن 
مؤسسات المعلومات من تقديم خدمات أكثر واسرع ، و افضل عبر الانترنت دون تحمل

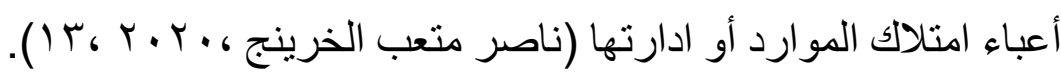

كما انها توفر للمستفيد ومؤسسات المعلومات امكانية تخزين المعلومات ومعالجتها وتناقلها و التشارك فيها من اى مكان وفى اي وقت دون الالتزام باستخدام الحاسب الثخصى، وانما يتم انجاز اجراءات (التخزين ، المعالجة ، التناقل و المشاركة ) فى لهى

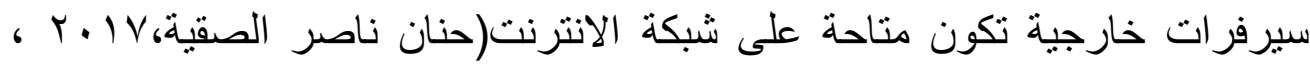
. $(r \leq r$

كما أنها تساعد على تحسين وتطوير خدمات المعلومات ، خاصة فى مجال التعليم كسهولة الوصول الى المعلومات المتو افرة على شبكة الانترنت و غير ها من شبكات المعلومات ،حيث تساهم استخدام تطبيقات الحوسبة السحابية بشكل كبير فى سهولة

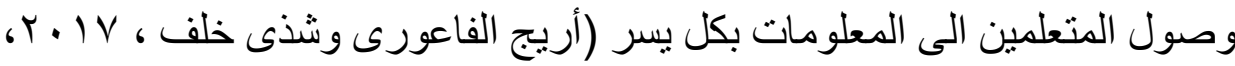
.$(194$

كما توفر للمعلمين تطبيقات لاعداد الاختبار ات ومشاركتها عبر الانترنت و التعامل مع المتعلمين من خلال ادارة المحتوى التعليمى الالكترونى، كتقييم الاختبارات، وارسال الواجبات و والمشاريع للمتعلمين واستلامها منهم كتغذية راجعة فورية.(kumar,2014,73) وقد اتاحت شركة جوجل خدماتها وتطبيقاتها السحابية،ووفرت سحابة جوجل التعليمية العديد من وتطبيق جوجل در ايف Google Drive التطبيقات السحابية مثل Gmail بريد جوجل .(Mansour, 2013, 12) 


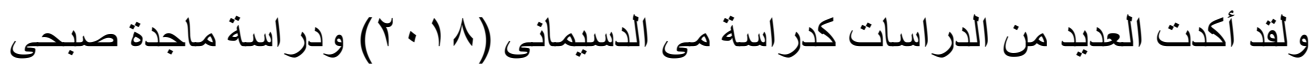
متولى (T (Y T) فاعلية البرامج القائمة على الويب فى تنمية المهارات والمعلومات وتطو ير ها،وفى تحصيل المعارف و اثر ائها وتجديدها .

كما أثنارت العديد من الدر اسات كدر اسة محمود سيد محمود(9 (ب Y) ودر اسة بشرى

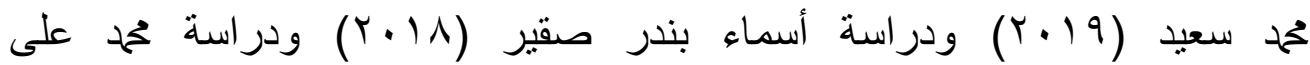

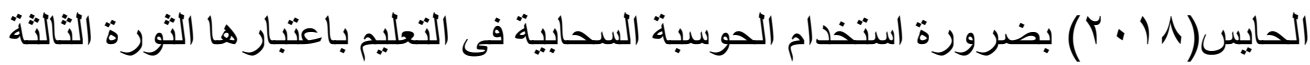
بعد كلا من الحاسب الالى وشبكة الانترنت ، بالاضافة الى وجود حاجة مستمرة لتنمية مهار ات القرن الحادى والعشرين التى تتطلب تفاعلا اكبر بين المعلمين والمتعلمين وتوفير ادوات تدعم تتمية مهار اتهم وهذا ما توفرة تطبيقات الحوسبة السحابية .

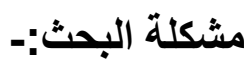

يعتبر الويب من اهم التطور ات المعاصرة ذات الدلالة فى العالم المعاصر ، كما ان التعلم القائم على الويب من اهم مستحدثات تكنولوجيا المعلومات والاتصالات و التى تمثل اولوية فى البحث والتطوير نظر اللتطورات المتسار عة، وتعد الحوسبة السحابية مهمة فى العملية التعليمية حيث ان بيئة الحوسبة السحابية نؤدى الى تعزيز التعلم وممارسة الانشطة واثر اء الخبرات التعليمية (Wettasing,2012) .

و على الرغم من اثـارة الدراسات الى العديد من مزايا الحوسبة السحابية التى تجعل منها تقنية فاعلة وملائمة للاستخدام فى مجال التعليم الا ان هناك قصور ا فى المهارات اللازمة لاستخدام تطبيقات الحوسبة السحابية مما ادى الى ضعف استخدام تقنية

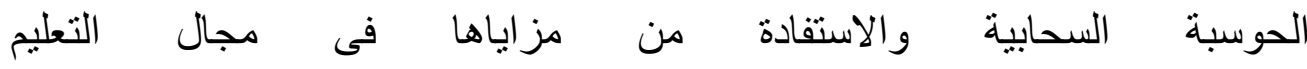
.(Bahga\&Madisetti,2013)

اسئلة البحث :- حاول البحث الاجابة على السؤال الرئيسى التالى : 
"ما فاعلية البرنامج القائم على الويب لاستخدام بعض تطبيقات الحوسبة السحابية فى التدريس لاى معلمى المرحلة الاعدادية ؟ هدف البحث :- هدف البحث التالى الى :

التعرف على فاعلية برنامج قائم على الويب لاستخدام بعض تطبيقات الحوسبة السحابية فى التدريس لاى معلمى المرحلة الاعدادية .

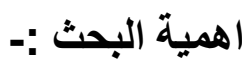

ا -قد يفيد البرنامج القائم على الويب المعلمين فى استخدام بعض تطبيقات الحوسبة السحابية فى التدريس.

Y-قد يساعد البرنامج فى نزويد المعلمين بنموذج عملى حول كيفية استخدام بعض تطبيقات الحوسبة السحابية فى التدريس. محددات البحث:- اقتصر البحث على الحدود الاتية : 1 ـالحدود المكانية : بعض مدارس مركز ارمنت بمحافظة الاقصر

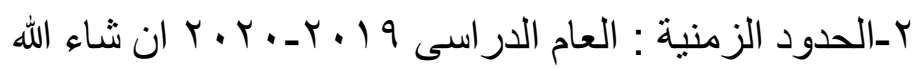
r-الحدود الموضوعية : يقتصر هذا البحث على تنمية مهارات تطبيقات الحوسبة السحابية

المحور الاول :التعلم القائم على الويب 
تعددت تعريفات التعلم القائم على الويب وتباينت وفقا لاراء الدارسين و الباحثين فمنهم من عرفه انه استخدام شبكة المعلومات العالميه فى تعليم الطلاب،حيث تعمل كمصدر اساسى من مصادر التعلم ،وكوسيط لتبادل المعلومات ،وحدوث التفاعل بين

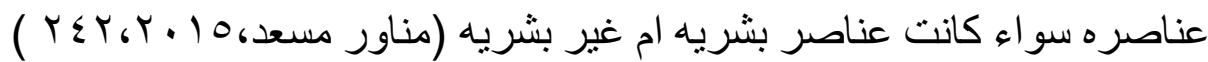
ـكما يمكن تعريفة بأنة :بأنه تقديم الدروس عبر الثبكات الحاسوبيه العامه او الخاصه

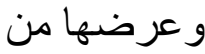

خلال متصفح الثبكة العالمية للمعلومات ( Simpkins, 2015,2) - ويعرف ايضا انه اتاحة برامج المقررات التعليمية عن بعد عبر صفحات الويب،

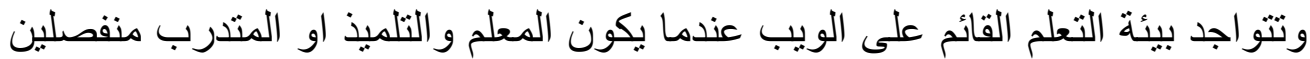

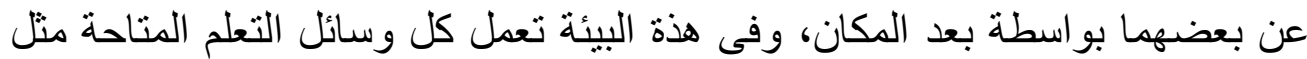
البيانات ولقطات الفيديو و الرسومات و الاشكال الثابتة و المتحركة و والمرئيات

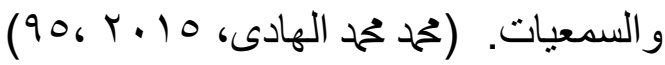

مما سبق يمكن تعريف التعلم القائم على الويب بانه : ذلك التعلم الذى يتم من خلال الوسائط الالكترونيه و الذى يعتمد على التقنيات و المستحدثات التكنولوجيه و الرقميه الحديثه والتفاعليه ،ويتمركز هذا النوع من التعلم حول المتعلم ويتجاوز حدود الزمان

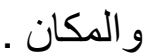

ثانيا :أهمية التعلم القائم على الويب

نظر ا لاهية التعلم القائم على الويب فى عمليتى التعليم والتعلم اهتمت به العديد من

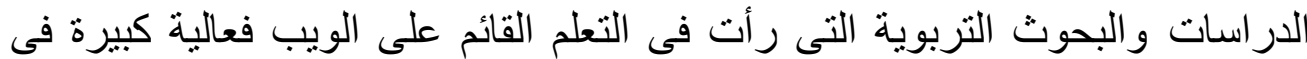

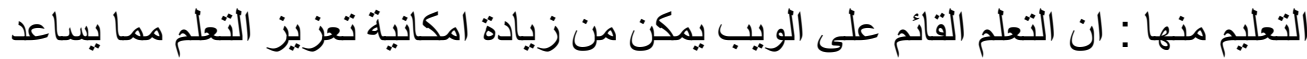

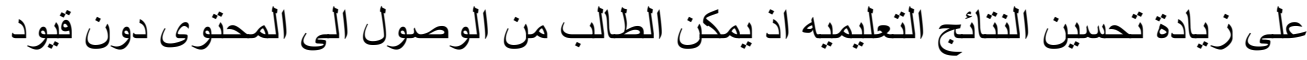

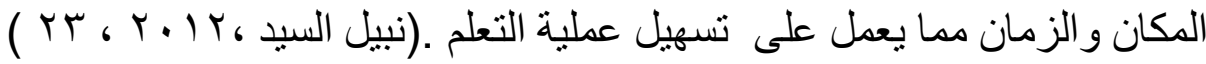
كما أن التعلم القائم على الويب يساعد على توفير الخدمات التعليميه والتعلميه بصورة افضل حيث يساعد على التعلم عن طريق المناقثاه البناءه وتبادل الخبرات 


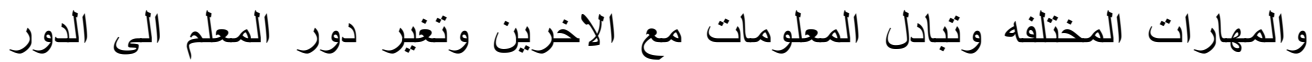

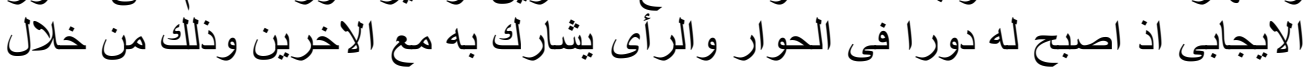

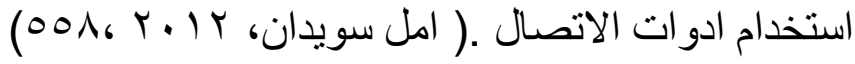

$$
\begin{aligned}
& \text { ثالثا: خصائص التعلم القائم على الويب }
\end{aligned}
$$

ان للتعلم القائم على الويب خصائص تميزه وتخصه عن غيره من اساليب التعليم

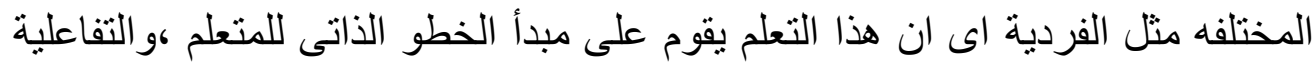

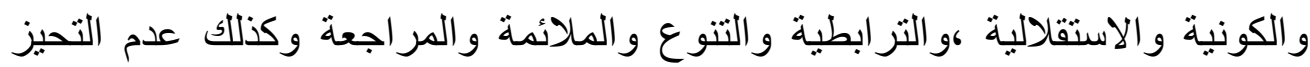

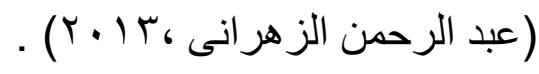

رابعا: مميزات التعلم القائم على الويب

من مميز ات التعلم القائم على الويب أنه يتميز بسهولة ومرونة تطويره وتحديثه ، ويقدم

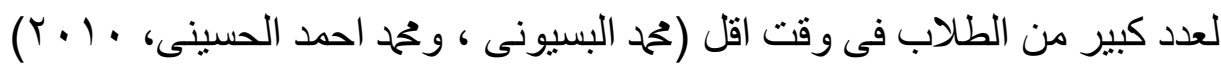
المحور الثانى : الحوسبة السحابية

اعتمدت العملية التعليمية ولقتر ات طويلة على الطرق التقليدية ،وركزت على المعلم كمصدر اساسى للمعرفة والمعلومات ،وفى ظل التطور التكنولوجى الداعم للتعلم التحلم القائم على الويب اصبح من الضرورى التوجة لرسم استراتيجية متميزة لاستخادم تقنية

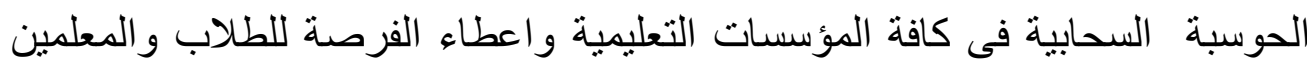
من الوصول السريع لمختلف النظم و التطبيقات.

اولا :مفهوم الحوسبة السحابية

هى تقنية ناثنئة توفر خدمات تكنولوجيا المعلومات و الموارد الى المستخدمين من خلال

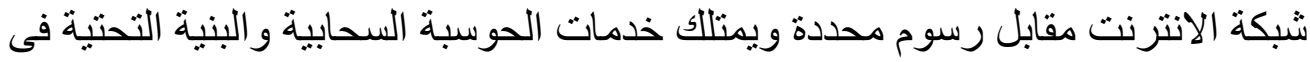

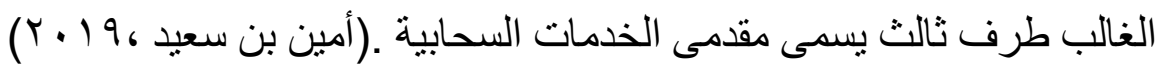

كما تعرف بأنها عبارة عن جهاز خادم يتم الوصول اليه عند الطلب عن طريق شبكة

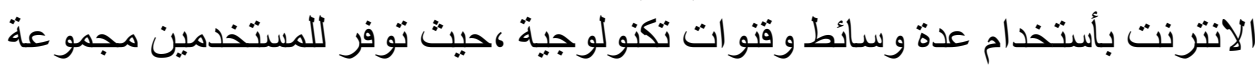

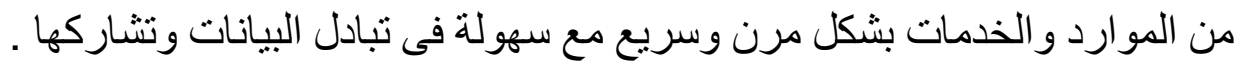

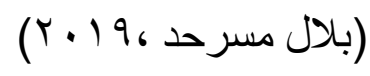


وتعرف أيضا بأنها الموارد و الانظمة الألكترونية المتاحة عبر الأنترنت و التى تستطيع

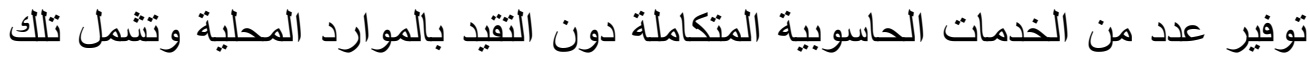

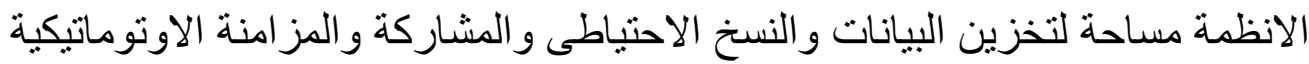

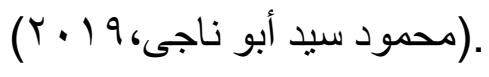

مما سبق يمكن القول ان الحوسبة السحابية هى تكنولوجيا حديثة تتم من خلال نقل عمليات معالجة المعلومات وتخزينها من حاسبات المستخدمين الى حاسب الى مركزى يتم الوصول اليه عن طريق الانترنت فى اى زمان ومن اى مكان .

\section{ثانيا : انواع الحوسبة السحابية}

اختلفت الدر اسات فى تحديد انواع الحوسبة السحابية ،فهناك در اسات حددتها بثلاثة

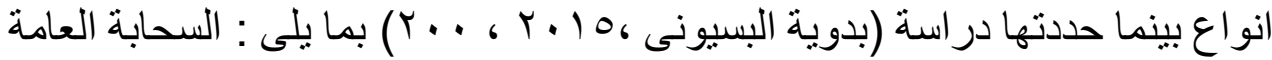
ـ السحابة الخاصة ـ السحابة المجتمعية ـ السحابة المختلطة ـ السحابة الشخصية ـ

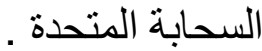

\section{ثالثا:مميزات الحوسبة السحابية :}

من اهم مميز ات الحوسبة السحابية انها تركز على انشطة التدريس و البحث العلمى بدلا من نظم البرمجيات المعقدة ،و امكانية استخدامها لمسـاندة طر ائق التدريس القائمـة على

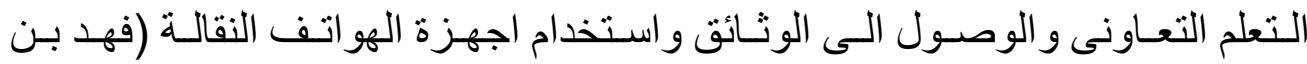

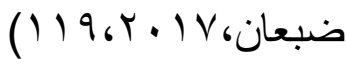

كما ان التوجـة نحو استخدام الحوسبة السـحابية حظى بتأييد عدد من النظريـات منهـا النظرية البنائية الاجتماعية و التى تنظر الى التعلم كنشـاط بنائى اجتمـاعى يعتمد على التشـارك الاجتمـاعى و التفاعل بين الافر اد بهدف انجـاز مهام تعليميـة وتقديم النظريـة الاتصـالية دعمـا متميـز ا للتعلم مـن خـلال بيئـة الحوسـبة السـحابية (زينـب حمحــ حسـن 


\section{المحور الثالث : تطبيقات الحوسبة السحابية}

تبذل المؤسسات التعليمية جها كبيرا فى البحث عن افضل النظم التعليمية من حيث التقنيات والسرعة والمرونة للحصول على تعلم اكثر فاعلية من خلال شبكة الانترنت،كما ان العديد من شركات تكنولوجيا المعلومات اتجهت الى اعتماد تطبيقات الحوسبة السحابية فى التعليم وعلى رأسها تطبيقات جوجل التعليمية ومن اهم تطبيقات Google Drive سحابة جوجل التعليمية تطبيقتيق

$$
\text { Gmail و البريد الالكترونى }
$$

ومن اهم الدراسات التى تناولت تطبيقات الحوسبة السحابية فى العملية التعليمية

$$
\text { واهميتها دراسة }
$$

-در اسة بشرى ححم سعيد (9 1 ب ب) التى تناولت اثر تطبيقات الحوسبة السحابية فى تنمية

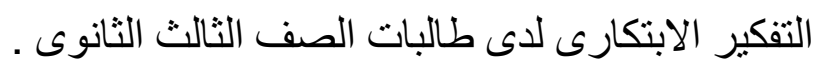

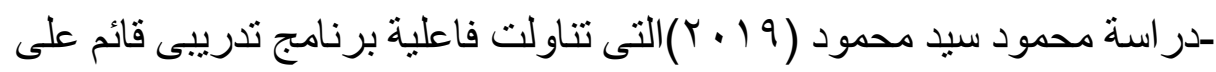
تطبيقات الحوسبة السحابية فى تنمية مهار ات ادارة المحتوى الالكترونى لدى لدى طلاب

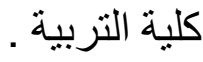

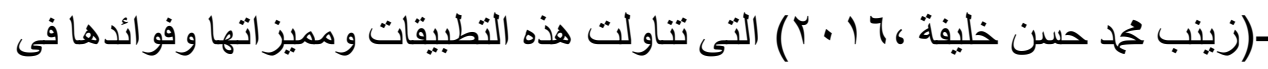
العملية التعليمية واوصت بالاستفادة من تطبيقات الحوسبة السحابية التى تتاح بصورة التهات كاملة مجانية. منهج البحث :

ينتمى هذا البحث الى فئة البحوث التى تستهدف اختيار العلاقات المسببة بين

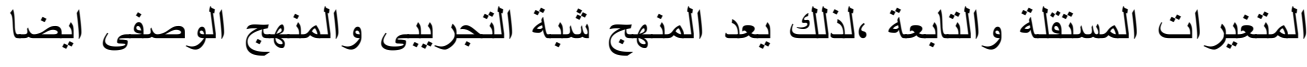

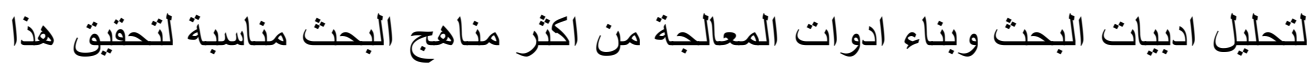

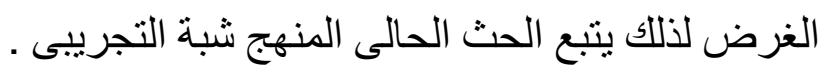


التصميم التجريبى للبرنامج

تم التصميم وفقا للخطوات التالية :المرحلة الاولى :

ا - مرحلة التحليل:تشتمل على (خصائص الفئة المستهدفة ، الاهداف، الموضوعات ،

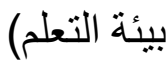

r _المرحلة الثانية مرحلة التصميم : وتتنمل هذة المرحلة على عدة خطو ات وهى : - تحديد المحتوى التعليمى للبرنامج ـ تحديد الاهداف العامة للبرنامج - تحديد الاهداف الاجر ائية لمحتوى -وضع سيناريو التعلم داخل البرنامج البرنامج

- تحديد الوسائط الرقمية المناسبة - تصميم انشطة ومهام التعلم المرحلة الثالثة :انتاج البرنامج

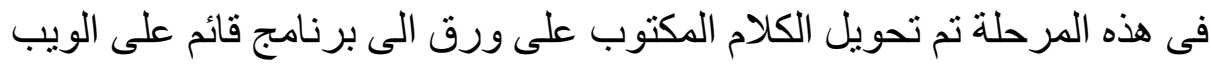

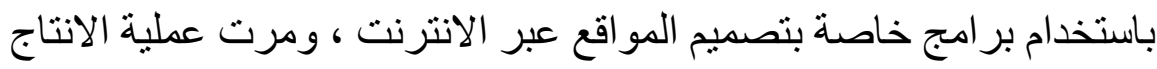

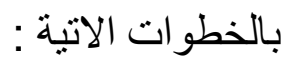

إـ اختيار بر امج التاليف Google Sites هو احد بر امج تصميم المو اقع المجانية

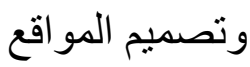

r-تحديد بنية التجول داخل الموقع لتوضيح عملية التنقل بين صفحات الموقع احتوت

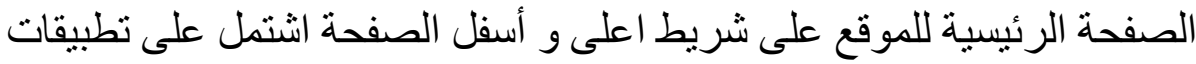

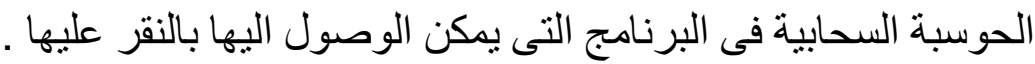

ץ- تصميم الصفحة الرئيسية للموقع هى صفحة البداية التى تظهر للمستخدم عند كتابة

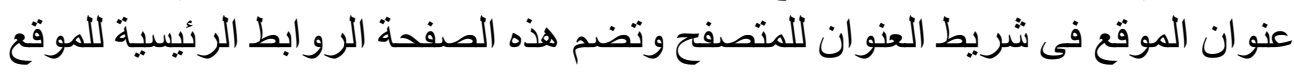

ـ - تصميم باقى صفحات الموقع من خلال : تحرير المحتوى بما يشملة من مهار ات

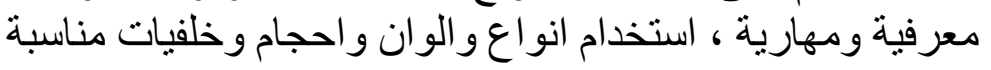
0ـ ربط الموقع بخدمات الثبكة انتاج النسخة التجريبية للموقع 


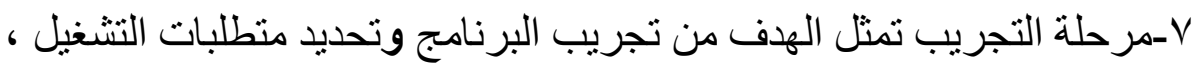
و عرض البرنامج على السادة المحكمين

المرحلة الر ابعة :نشر وتنفيذ البرنامج وتشتمل هذه المرحلة على نشر و استخدام

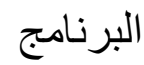

\section{نتائج البحث}

من خلال المعالجة الاحصائية واستخدام برنامج (Spss) للمعالجة الاحصائية لمقارنة

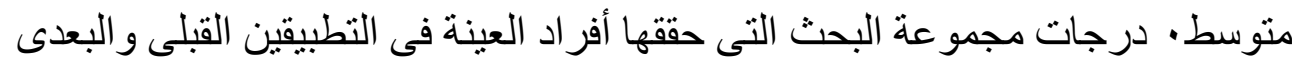

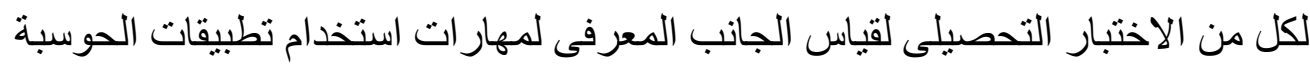
السحابية فى التدريس ،وبطاقة الملاحظة لقياس المهار ات العملية ،وبتحليل النتائج تبين ما يلى :

ـاظهرت نتائج التطبيق القبلى لادوات البحث ان معلمى المرحلة الاعدادية (مجموعة

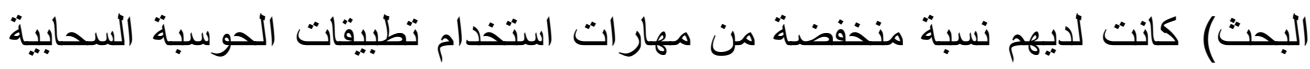

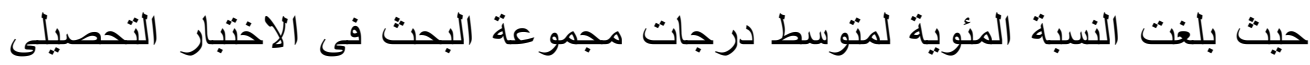

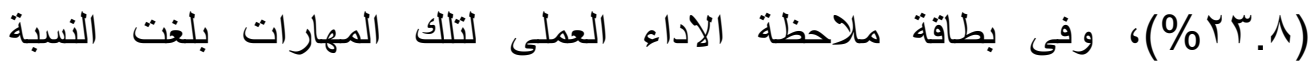

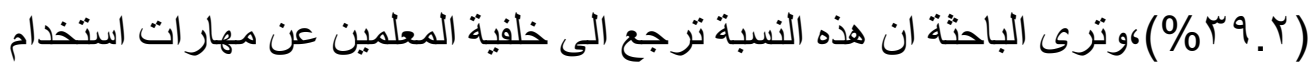

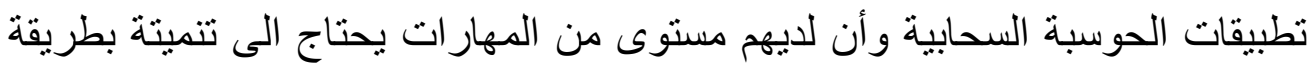
تتناسب مع قدر اتهم العقلية وكذللك ظروفهم المعيشية والبية لائية .

ـاظهرت نتائج التطبيق البعدى للاختبار التحصيلى لقياس الجانب المعرفى لمهارات

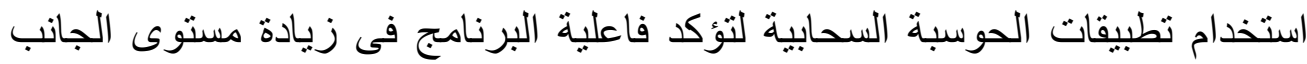

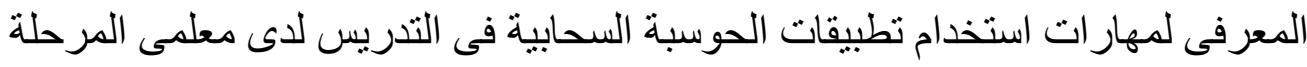

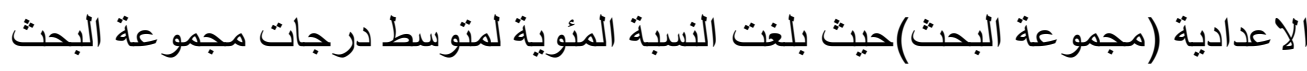

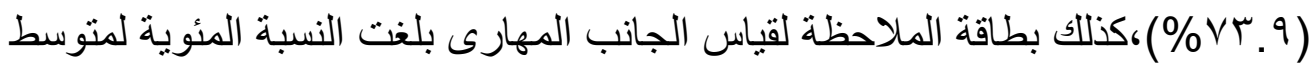

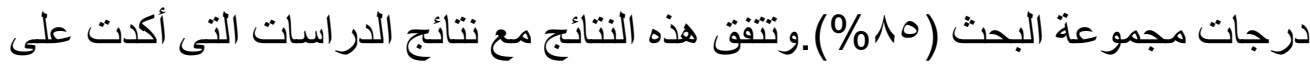

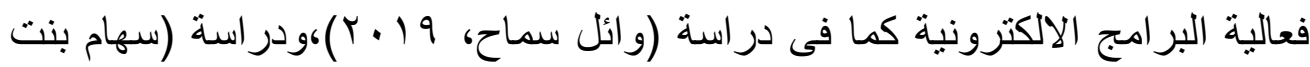

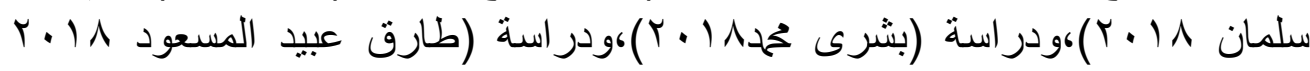

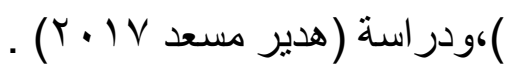


ويمكن ارجاع ذلك الى ما يلى :

1 ـاحتو اء البرنامج القائم على الويب على الوسائط المتعددة و المعلومات الو اضحة

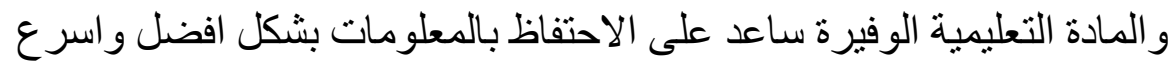
Y-قدرة البرنامج على توفير فرص للمعلمين على التركيز والانتباة

r-بناء البرنامج على مبادئ النظرية البنائية التى تجعل المتعلم محور العملية التعليمية ع-مساهمة البرنامج فى زيادة دافعية المعلمين على حب التعلم و الاقبال على تعلم

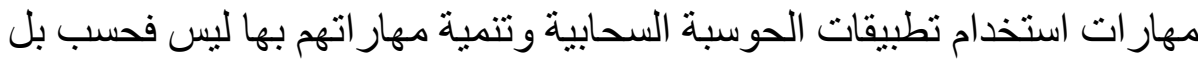
وتوظيفها فى طر ائق تدريسهر

توصيات البحث :فى ضو ء نتائج البحث يمكن وضع التوصيات التالية : ا ـالعمل على تو عية المعلمين فى المدر ارس بضرورة استخدام تطبيقات الحوسبة

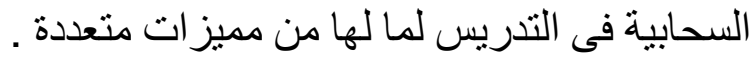

ب-تضمين المناهج الدر اسية فى مر احل التعليم المختلفة بمعلومات عن تقنية الحوسبة

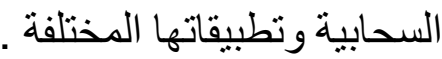

ب-توجية اهتمام القائمين على انتاج البر امج التدريبية الى تصميم بر امج تدريبية قائمة على بعض تطبيقات الحوسبة السحابية .

ـ ـ-عمل دور ات تدريبية للمعلمين للتدريب على استخدام تطبيقات الحوسبة السحابية ج-تو افر الرسوم التوضيحية والارشادات و الصور داخل البرنامج ساعد على ارتفاع

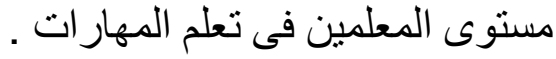




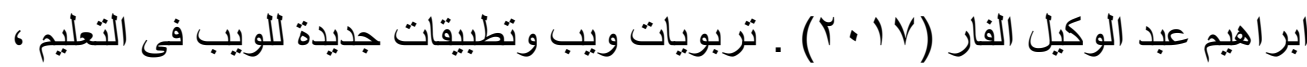

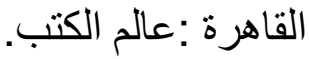

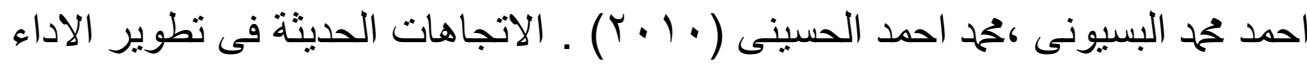

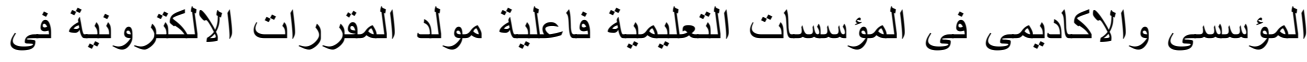

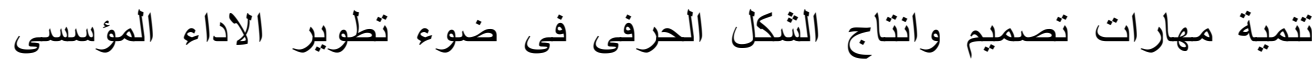

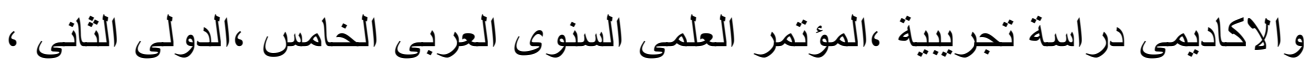

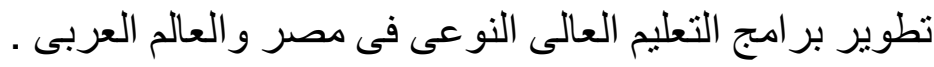

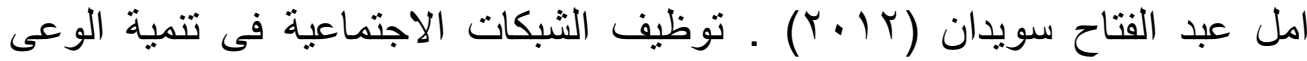

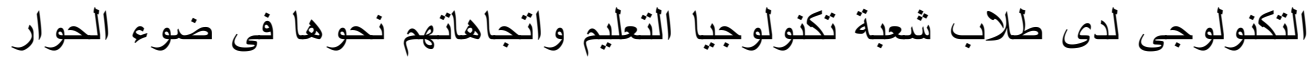

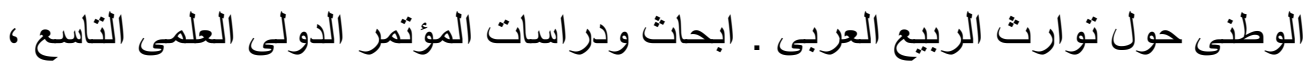

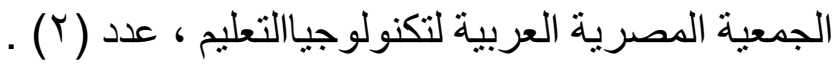

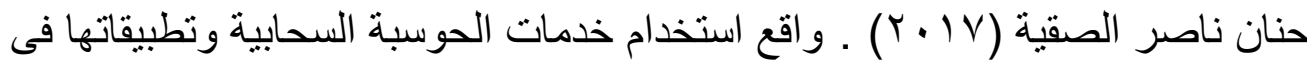

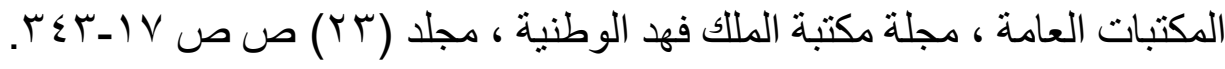

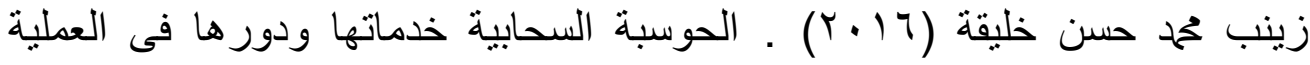

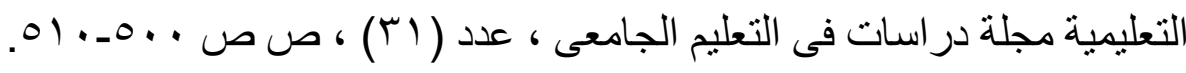

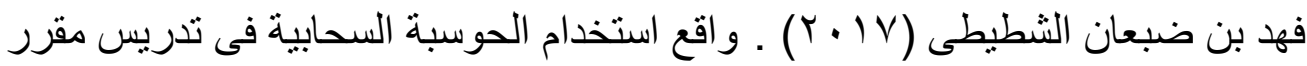

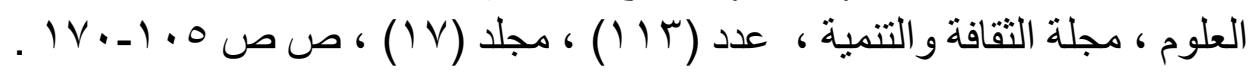

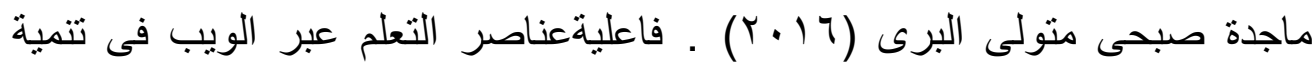

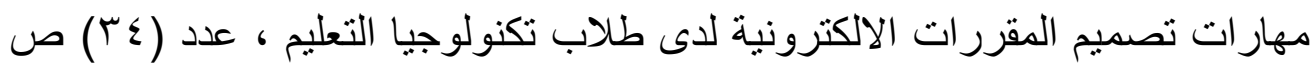

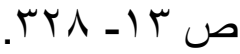

عحمد الباتع ححمد عبد العاطى (10 ب ب) ـ توظيف تكنولوجيا الويب فى التعليم ، القاهرة :

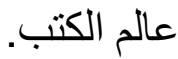

،حمد محمد الهادى (10 ب ب) ـ التعليم الالكترونى عبر شبكة الانترنت ، افاق تربوية مجددة ، القاهرة : الدار المصرية اللبنانية. 


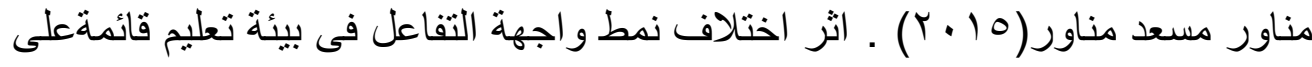

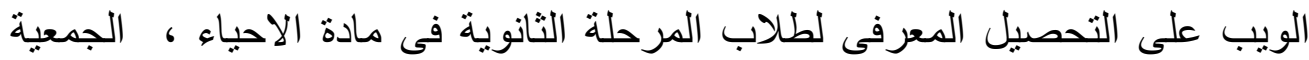

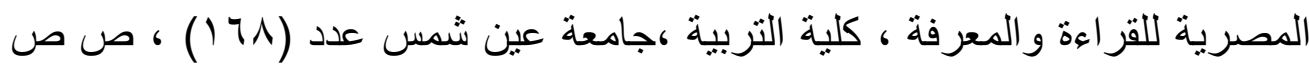
. $Y \leq Y-Y \leq$.

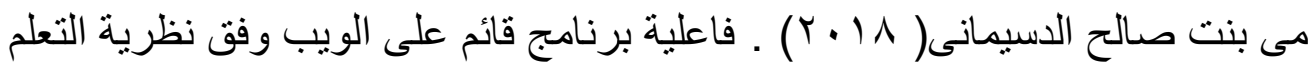

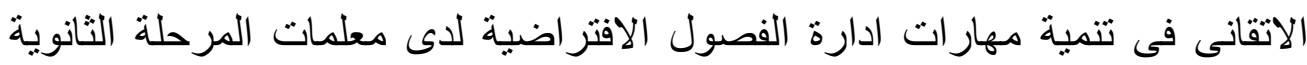

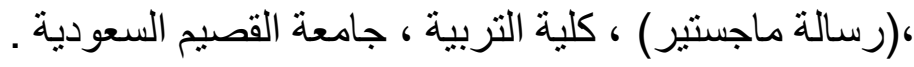

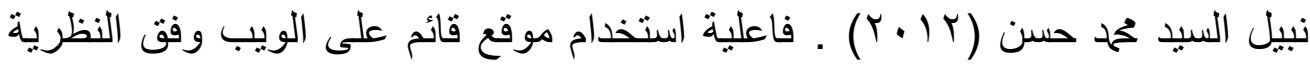

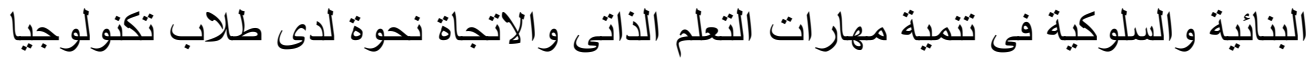

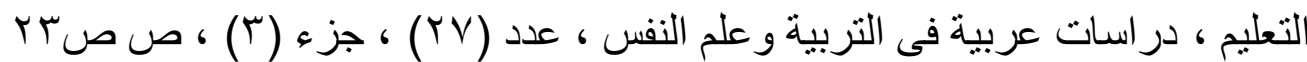
rv-

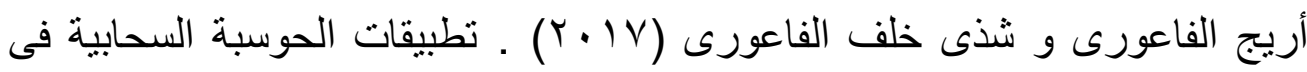

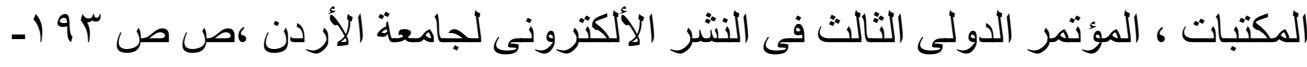

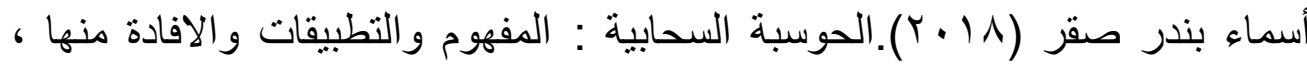

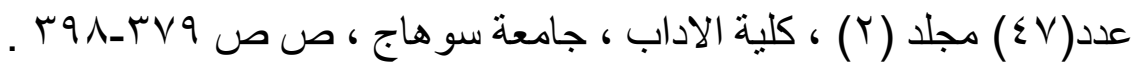

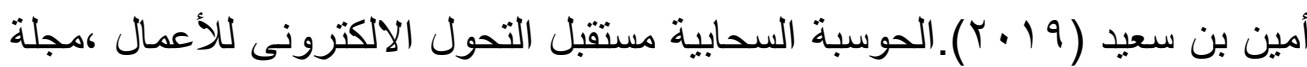

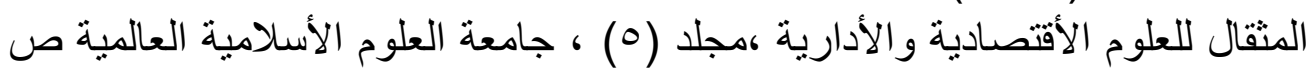
ص ص ال

بدوية حمحد البسيونى (10 (Y)). برمجيات الحوسبة السحابية ودور ها فى تطوير خدمات

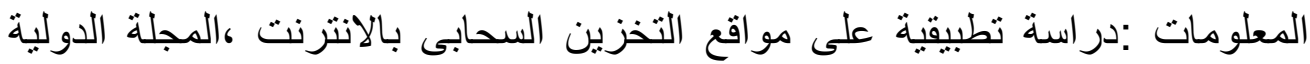

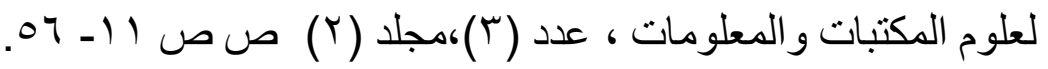

بشرى محمد سعيد الزهرانى (9 (19). أثر بيئة الحوسبة السحابية فى تنمية التفكير

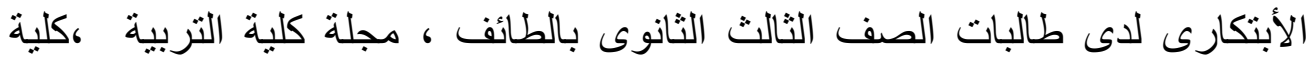

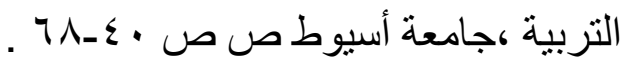


بلال مسرحد (9 (1).تصور حكومة الحوسبة السحابية فى المؤسسات الحكومية

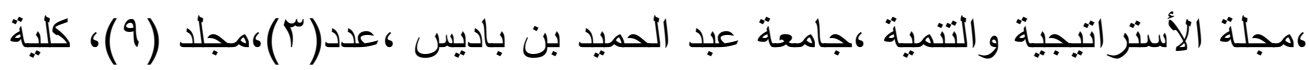
العلوم الاقتصادية .

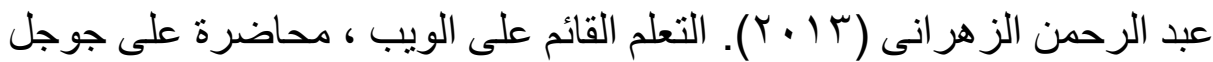
در ايف ، متاح على الر ابط الر هري http://drive.google.com/file/d/0b6qefekujfvnvuvvuujmlu82vuk /view?usp=sharing

محمد على الحايس(1 ( ـ ب).برنامج مقترح قائم على تطبيقات الحوسبة السحابية لتنمية

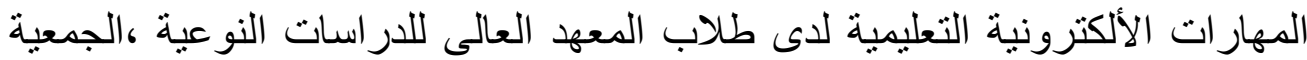

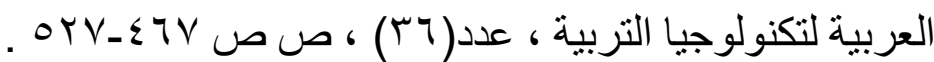

محمود سيد محمود أبو ناجى (19 (Y) ) . برنامج تدريبى قائم على تطبيقات الحوسبة السحابية وفاعليتة فى تتمية مهار ات ادارة المحتوى الألكترونى ،مجلة كليد كلية التربية

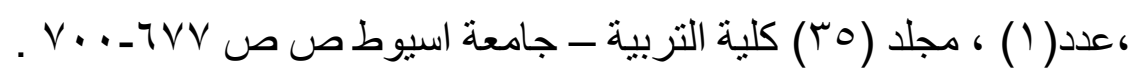

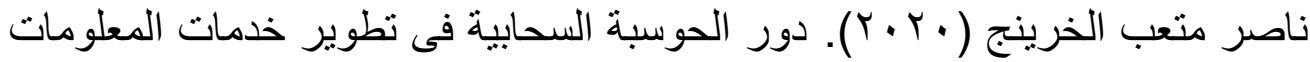
فى المكتبات الأكاديمية :دراسة مقارنة ، المجلة العلمية للمكتبات و الوثائق و المعلومات

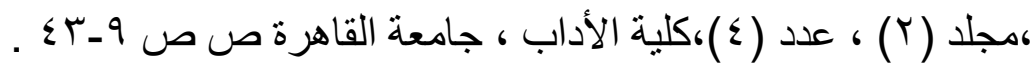

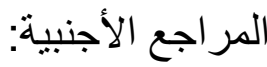

Simpkins, B.K.(2015) Homeland Security Training Transfer : Does Training delivery Method Matter ? Doctoral dissertation, Eastern Kentuck University, United States, Kentucky .

Kumar, R. (2014). Cloud Computing in Elearning for Different Perspectives of Teacher Education . International Jornal of Multidisciplinary Research and Development, 1,(6) 
Mansour, A, (2013). The Adoption of Cloud Computing Tachnology in higher Education Institutions: Concerns and Challenges (Case Study on Islarnic University of Gaza

Wettasing, R. (2012). Check the Status of Secondary Schools that tried Integrating the Traditional Method and the Use of Computhing Applications in ataeaching And Learning. Master theis, Providence University, Taiwan.

Bahga,A.\& Madisetti, V. (2013).Using Google Drive Tofacilitate a blended approach to authentic learning .british journal of educational technology 44 (4) 594-606 